\title{
Comparative Analysis of Various Energy Efficient Protocols for Wireless Sensor Networks
}

\author{
Jasdeep Kaur \\ Dept. of Computer Science \\ Guru Nanak Dev University, \\ Amritsar, Punjab, India
}

\author{
Sandeep Wraich \\ Dept. of Computer Science \\ Guru Nanak Dev University, \\ Amritsar, Punjab, India
}

\begin{abstract}
Due to limited battery of sensor nodes, energy efficiency becomes very critical issue. This paper presents a comparative analysis on various protocols of Wireless sensor networks. The overall objective of this paper is to evaluate the best protocol among the available protocols. This paper has shown that the still majority of research on WSNs suffers from certain issues. The use of the hard and soft thresholds has been ignored by the most of the existing researchers. In many protocols node might not become cluster head for a long time so will result in load misbalancing. The comparative analysis of the protocols have been done with the parameters based ondata fusion, energy aware, route selection, base station, inter-cluster and intra-cluster. This paper has shown that the protocol BEEM has found to be very effective among others.
\end{abstract}

\section{Keywords}

WSNs , Energy, Thresholds, Network Lifetime.

\section{INTRODUCTION}

A Wireless Sensor network is a set of sensors, deployed in a sensor field to monitor specific characterization of the environment to measure those characteristic and collect the data related to the phenomena in which sensors are small devices with limited resources such as limited battery power, low memory, little computing capability, very low data rates, low bandwidth processing, variable link quality [11]. Sensor nodes are defined by two categories: First one is called Normal node, which is used to sense phenomena and Second node is called Gateway node that is used to act as interface between sensor network and external world.The absence of wires implies the lack of an external power supply such as battery packs. Therefore it is necessary to extend the battery life of individual sensors so that the network can remain functional as long as possible.In WSNs we have to look at the network topology from a different perspective, from a neighborhood point of view. In these topologies, the number of neighboring nodes determinesthe number of receivers and hence results in more overall power usage, even though the number oftransmissions decreases. Thus, there is a fundamental trade-off between decreasing the number of transmissions and increasing the number of receptions.WSN use Routing as for data transfer between nodes. Sensor nodes send their data to corresponding cluster head nodes which aggregate data and send to base station either directly or by intermediate cluster head nodes. Base station is point where data is received from sensor nodes and accessed by user. Cluster head act as the interface between the sensor node and base station.

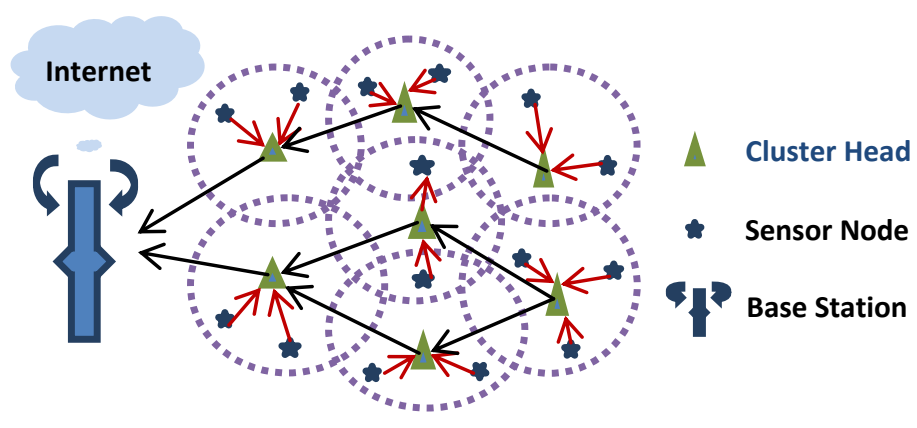

Figure 1: Wireless Sensor Network Model

WSN networks are data centric networks. They focus on what is the data rather than where is the data.WSN is used in many real time applications such as Transportation and Logistics, Smart buildings such as indoor climate control, Smart grids and energy control system, Health care such as health monitoring and medical diagnostics, Security and surveillance, Entertainment, Urban terrain tracking and Structure monitoring, Environmental monitoring, Precision agriculture and animal tracking, Industrial applications. Clustering in Wireless Networks is the process of dividing the nodes of network into different groups where each group agrees on center node which is known as cluster head. Cluster head is responsible for collecting the data of group members and aggregate that data and send this data to the base station.Some of the Clustering approaches are TEEN[18], APTEEN[17] , M-LEACH[14] ，SEP[7] ,TL-LEACH[12], LEACH-C[16] , BEEM[2] , NEW-LEACH[5], HEED[13], EECH[15], T-LEACH[8], LEACH [1]. In Clustering process, Selection of cluster head plays an important role in performance of network. Selected cluster head should have equal distance from sensors own cluster, gather sensors and have enough power for sending information to the base station. Geographic routing algorithms are used for twodimensional (2D) networks. Geographic routing is of interest for sensor networks because a point-to-point primitive is an important building block for data-centric applications. Many methods have been used to obtain better cluster by using geometrical shapes such as 2D (Two dimensional) and 3D(Three Dimensional)[9]. It is assumed that all nodes of network reside on a plan in 2D design of terrestrial network. This assumption is not always valid if a network is deployed in space or ocean, where nodes of network are distributed over 3D space. 


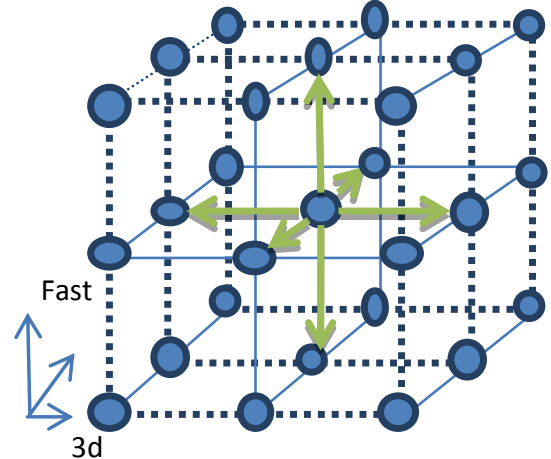

Figure 2: 3-dimensional environment

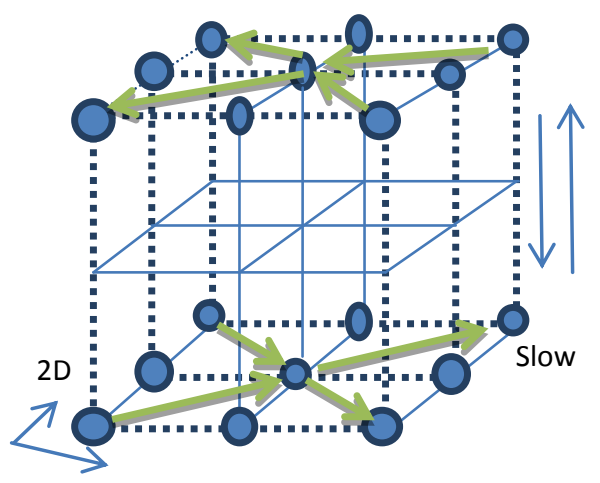

Figure 3:2-dimensional environment

Many designs have been made in 2-dimansional (2D) space but review space is always a surface. To resolve this restriction 3-dimensional (3D) clustering is introduced. The design of 2D wireless network has been mostly considered in recent research approaches, while the 3D wireless sensor network has not been so much explored. In reality, most wireless sensor network operate in three dimensional. 3Dimensional scenario of WSN represents more accurately the design of sensor network for real world applications. Three Dimensional shapes such as cube, pyramid or cylinder are used. These shapes have different review for surface that are monolithic and dependent on each other. As in pyramid shape we have three levels known as Root level, Middle level and Lowest level. Sink is placed at the root level, which receive data from lower nodes. Cluster head is placed at the middle level that is in charge of receiving data and manage the transmission process fromactive nodes to root node. Lowest level is known as the Active node level, in which the numbers of nodes that are all active are grouped into some cluster according to the above cluster head.

\section{TAXONOMY OF CLUSTERING PROTOCOLS}

\subsection{LEACH (Low energy adaptive clustering hierarchy)}

Leachselects randomly the nodes cluster heads and allocate role to different nodes by using round robin policy to ensure fair energy dissipation between nodes. In order to minimize the amount of information sent to base station ,the cluster head aggregate the data collected by the member node related to their own cluster and then on aggregate packet to the base station. Leach protocol perform operation in two steps. First step is called Set-up Phase. In setup phase, clusters are organized from network structure. In this step every node makes decision aboutto be a cluster head or not asa Cluster Head. This decision is depend on the proposed percentage of Cluster Headto the network and the cluster node has become a cluster head for how many number of times. The decision of becoming a cluster head is made by the node selectingaarbitrary number between 0 and 1 . The sensor node will turn to a Cluster Head if the number is not more than the following threshold [1]:

$$
T(n)= \begin{cases}\frac{P}{1-P\left(r \bmod \frac{1}{P}\right)} & \text { if } n \in G \\ 0 \quad & \text { otherwise }\end{cases}
$$

Where $P$ is the desired percentage of $\mathrm{CHs}$, is the current round, and is the set of nodes that have not been elected $\mathrm{CHs}$ in the last $1 / P$ rounds [1]. The second step is called SteadyState Phasewhere data is delivered to the Base Station and compressing sensing of data is done.

\subsection{HEED (Hybrid Energy Efficient Distribution protocol)}

This protocol is a multi-hop Wireless clustering protocol which causes an energy-efficient clustering routing with direct reflection of energy. Heed is diverse from LEACH in the manner of $\mathrm{CH}$ selection, cluster heads are not selected randomly.In HEED, Cluster Heads are selected by two parameters as residual energy and intra-cluster transmission cost of the nodes. We assume $C_{\text {prob }}$ is an optimal percentage that cannot be computed previously. The probability that a node becomes a $\mathrm{CH}$ is [13]:

$$
C H_{\text {prob }}=C_{\text {prob }} \frac{E_{\text {residual }}}{E_{\text {max }}}
$$

Where $E_{\text {residual }}$ is the estimated current energy of the nodeand $E_{\max }$ is a reference maximum energy, which is normally identical for all nodes in the network [13].

The value of $\mathrm{CH}_{\text {prob }}$, however, is not allowed to fall below a certain threshold that is selected to be inversely proportional to $E_{\max }[13]$.

\subsection{TL-LEACH (Two-Level Hierarchy LEACH)}

This protocolexceeds Leach algorithm by performing routing in two level hierarchies. In this protocol [12], a Cluster Head gather data from Nodes as LEACH perform, but it does not send data to the Base Station directly, instead it uses a portion of Cluster head that is placed between the $\mathrm{CH}$ and the $\mathrm{BS}$ and named as a relay station. $\mathrm{CHs}$ at top position called as primary cluster heads $\left(\mathrm{CH}_{i}\right)$, second level $\mathrm{CHs}$ presented from secondary cluster heads $\mathrm{CH}_{i j}$ and ordinary Nodes. The algorithm can be defined by from four basic steps.First step is called Advertisement phase, where each node make decision about it will become a primary $\mathrm{CH}$, secondary $\mathrm{CH}$ or Ordinary Node in every round same as in LEACH protocol. If node decides to become a primary $\mathrm{CH}$ then node advertised other nodes by using carrier sense multiple access (CSMA).In second step which is called as Cluster setup phase, each secondary $\mathrm{CH}$ make decision that to which primary Cluster head this node wants to belongs and transmit signal as an advertisement message to its primary $\mathrm{CH}$. According to above technique every ordinary node should select which secondary cluster head this node wantsto belong and send this information by using opposite message. In the third step 
which is called as Schedule creation, every primary cluster head generates a TDMA schedule allocating every node in their sections asa time slot to transmit. Every primary cluster head selects a CDMA code and send message to all nodes at second level in its group to use code and by using above method each secondary cluster head can send this information to ordinary node in its cluster through both the code and the schedule from the primary $\mathrm{CH}$. In the fourth step which is called as Data transmission phase, clusters are generated and each node can send by using TDMA schedule organized by its primary $\mathrm{CH}$.

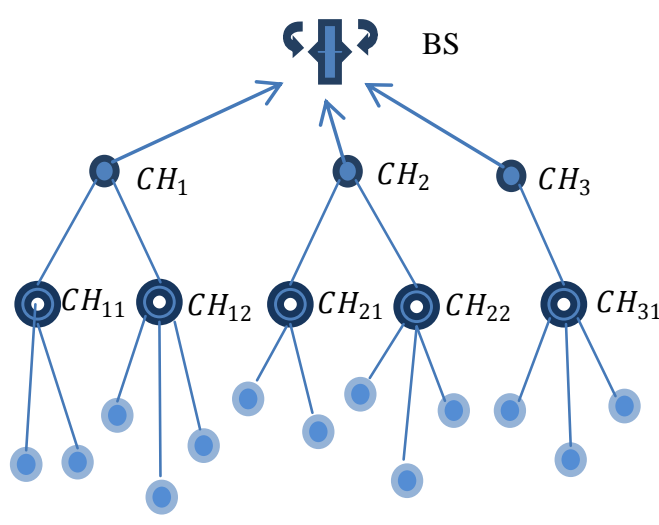

Figure 4: TL-LEACH

\subsection{TEEN (Threshold sensitive Energy Efficient sensor Network protocol)}

This is a hierarchical protocol whose main aim is to deal with unexpected changes in the sensed attributes such as energy. This protocol merges the tree based technique in line with a data-centric technique. The nodes absorb their environment stably, but the energy consumption in TEEN can probably be much less than that in the proactive network, due to reason that data transmission between nodes is done in less frequent manner. TEEN performs operations by defining two threshold functions [18]

Hard Threshold: This threshold is a threshold function value for the sensed element. It is the absolute value through which the node that has sensed this value must transfer to its transmitter and inform to its $\mathrm{CH}$.

Soft Threshold: The soft threshold is a small change in the value of the sensed attribute which causes the node to transfer to its transmitter.

In TEEN protocol, a $\mathrm{CH}$ transmits its nodes a signal of hard threshold and a soft threshold. The hard threshold tries to minimize the data communications by permitting the nodes to send only when the sensed attribute is in the range of sending area. The soft threshold minimizes the data communications that might have occurred when there is small or no change in the sensed attribute.

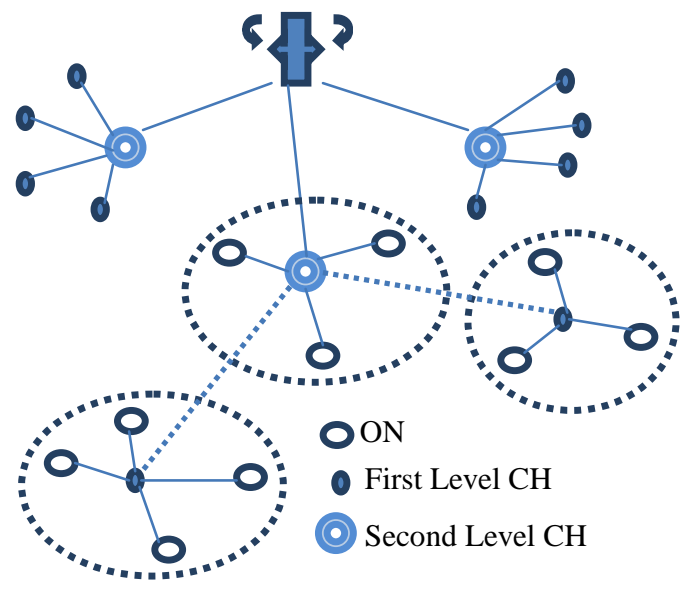

Figure 5: TEEN

\subsection{APTEEN (The Adaptive Threshold sensitive Energy Efficient sensor Network protocol)}

This protocol exceeds TEEN and main goal is to transmit both periodic data and reacting to time critical events. APTEEN is a hybrid protocol that changes the periodicity or threshold values used in TEEN as necessary according to customers and the type of the application required. APTEEN is defined by query based system thatpermits three types of queries: Historical, One-time, and Persistent. In APTEEN protocol, sink node broadcast the following four parameters [17]:

- Attributes: Theseare the set of physical variablesthat the customer is focused in acquiring data.

- Threshold functions: These functions composed of the hard threshold and soft threshold. Hard Threshold is a value that is used to trigger a node to send data to the sink node. On the other hand, Soft Threshold is a little change in the value that triggers a node to send data again.

- Schedule: Time Division Multiplexing schedule is used for distributing a slot to each node.

- Count time: Thisperiod is the maximum time limit between two regular outputs sent by a node. It can be a multiple of the TDMA schedule length and it can also accounts for the proactive attributes.

\subsection{EEHC (Energy Efficient Hierarchical Clustering)}

In this protocol every sensor node in the network becomes a cluster headwith probability $p$ and sends information to other nodes such that this node is cluster head within the range of radio. These cluster heads are known as the volunteer cluster heads. This information is transmitted further to all the nodes that there is no more than $k$ hops away from the cluster-head. Any sensor node that gets such information and is not a cluster-head in the given range, selects the cluster of the closest cluster-head. Any sensor node that is nota cluster head or has notchooses any cluster will becomes a cluster head. These type of clusters are known as the forced cluster heads. As we have boundedthe information sending to $k$ hops, then if a sensor nodeis not able to found $\mathrm{CH}$ transferring information within time slot $t$, it can conclude that it is not in the range of $k$-hops of other volunteer cluster head and willcome out to be a forced cluster-head. The bounding on the number of hops 
thus permits the cluster head to organize their communication.As supposed, the nodes are dividedaccording to a corresponding dimensional Poisson process and hence,the number of node in a square of side $2 a$ is a Poissonrandom variable, $N$ withmean $\Delta$ Awhere $A=4 a^{2}$. Let suppose that for a special awareness of the process there aren sensor nodes. Also assume that the treating center is at the center of the square. The probability of becoming acluster-head is $p$. Hence, on average, $n p$ sensors will become cluster-heads. Let $D_{i}$ be anarbitrary variable that denotes the length of the segment from a sensor located at $\left(x_{i}, y_{i}\right)$ where $i=$ $1,2,3, \ldots . . n$ to the handling center. Without loss of generality, we suppose that the processing center is located at the center of the square area. Then [15],

$$
E\left[D_{i} \mid N=n\right]=\int_{A} \sqrt{x_{i}^{2}+y_{i}^{2}}\left(\frac{1}{4 a^{2}}\right) d A=0.765 a
$$

Since there are on an average $n p \mathrm{CHs}$ and the location of any $\mathrm{CH}$ is independent of the locations of other $\mathrm{CHs}$, the total length of the segments from all these $\mathrm{CHs}$ to the processing center is $0.765 n p a$.

\subsection{SEP(Stable Election Protocol)}

A sensor network is measured which is hierarchically clustered similarto the LEACH. In wireless sensor networks, the clusters are recreated in everyround. Every round containsthree stage phases. The first stage is called as Cluster formation phase. In this stage clusters are arranged where as in second stage which is called as Virtual cluster head(VCH) formation phase, virtual cluster head is chosen by the selection condition. The third stage is called as Data transmission phase where data issent from the sensor nodes to the head node and passed to the sink node. New cluster heads are chosen in every round and asan outcome the load is spread and balanced between the nodes of the sensor networks. This protocol dependsupon original energy of node. This technique is used to allocate a weight to optimal probability $P_{\text {opt }}$. This weight should be equal to ordinary initial energy of every node distributed bythe initial energy of ordinary node. Let us supposed $P_{\text {ord }}$ is the weighted chosen probability of ordinary node and $P_{a d v}$ is the weighted chosen probability of other nodes.

$$
P_{\text {ord }}=\frac{P_{o p t}}{1+\alpha m}, \quad P_{a d v}=\frac{P_{o p t}}{1+\alpha m} \cdot \alpha(1+\alpha)
$$

Threshold function for ordinary node and advanced node [7]:

$$
\begin{gathered}
T\left(s_{\text {ord }}\right)= \begin{cases}\frac{P_{\text {ord }}}{1-P_{\text {ord }}^{*}\left(r \bmod \left(\frac{1}{P_{\text {ord }}}\right) \cdot\right)} & \text { if } s_{\text {ord }} \in G^{\prime} \\
0 & \text { otherwise }\end{cases} \\
T\left(s_{a d v}\right)= \begin{cases}\frac{P_{a d v}}{1-P_{a d v}^{*}\left(r \bmod \left(\frac{1}{p_{\text {adv }}}\right) \cdot\right)} & \text { if } s_{a d v} \in G^{\prime \prime} \\
0 & \text { otherwise }\end{cases}
\end{gathered}
$$

\subsection{NEW-LEACH}

This protocol is used to attain better result such as low energy consumption and longer lifetime through 3D environment in wireless sensor networks in different ways. Advanced sensor nodes are put into wireless sensor networks which have larger initial energy than the ordinary nodes. In this protocol when the cluster head is elected in every round randomly then the factors like residual energy, energy consumption rate and location information are considered. Consumption rate can be defined as the rate at which we find that how fast the energy of node is absorbed.In NEW-LEACH, the threshold function which is used to select the cluster head is defined as follow [5]:

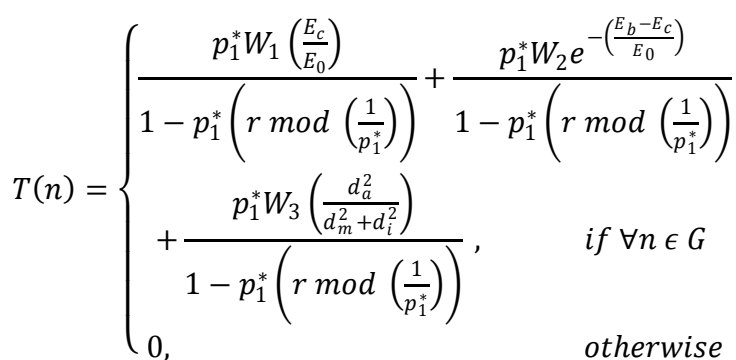

If the energy absorbed by the node is very fast then it is not realistic to be elected this node as the cluster head. But by composing the EEHC with this protocol, we can determine the optimal probability of node to become the cluster head in the wireless sensor network. Because of this reason the cluster head that is placed at distance from sink node consumed more energy directly from sink node than the node which is nearly connected to the sink node. New-Leach uses Hybrid routing scheme for the communication between the nodes. Where $E_{c}, E_{0}, E_{b}$ is the current energy, initial energy and the energy in the next round equating to the current round of the sensor node, $d_{a}$ is the average distance from the sensor nodes to sink, $p_{i}$ is the optimal probability of node to be a cluster head, $d_{m}$ is the maximum distance from the sensor nodes to the sink, $d_{i}$ is the distance of the current elected node $\mathrm{i}$ to the sink and $W_{1}, W_{2}, W_{3}$ is the weight which determine the threshold value as residual energy, energy consumption rate, location information respectively.

\subsection{LEACH-C (LEACH-Centralized)}

Leach protocol does notgive assurance about the placement of cluster head nodes in wireless sensor network. But by using a centralized technique to form the clusterscangenerates better clusters by scattering the cluster head nodes all over the network. This technique is the base for LEACH-Cprotocol that practices a central clustering algorithm and the similar steady state phase as in LEACH protocol. For the duration of Setup stage every node transmit information about its current position and also describe energy level to the base station, where cluster heads and theirrelated clusters are found. Base station announcesainformation that consists the cluster head ID for every node [16]. If the Cluster head ID equals to its own cluster head ID then the node become cluster head if not then node calculate its TDMA slot for data communication and goes to stable modeup to it is time to send data. 
Table 1: Comparison of different clustering protocol

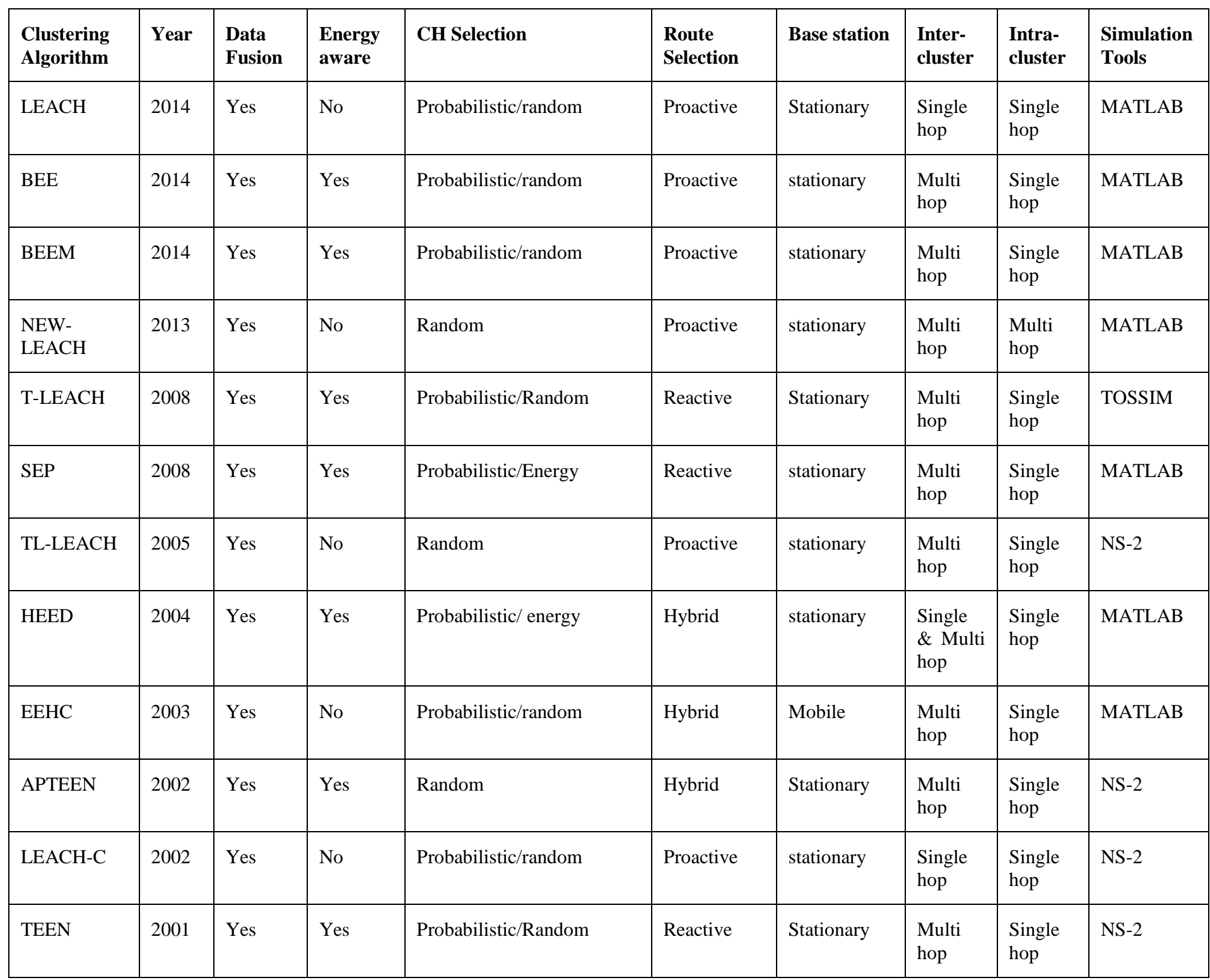

\subsection{BEEM (Balanced Energy Efficient Multi-hop Clustering)}

It is the algorithm based on HEED. BEEM exceeds HEED and LEACH from two perspectives that is longevity and balanced sensor distribution. It can guarantee the network coverage for a longer time, compared with HEED and LEACH. In BEEM nodes can communicate symmetrically. All the nodes are deployed randomly and placed at fixed location. All the set of nodes has same capability that is nodes are homogeneous in nature. The probability of sensor node to become a Cluster head can be calculated as [2]:

$$
C H_{\text {prob }}=C_{\text {prob }} \times \frac{E_{\text {residual }}}{E_{\max }} \times \min \left(\frac{\text { Node Degree }}{D_{\text {avg }}}, 1\right)
$$

Where $D_{\text {avg }}$ is the average density of network and calculated as:

$$
D_{\text {avg }}=\pi R^{2} \times \frac{\text { Num }}{\text { sensors }}
$$

Where $\mathrm{R}$ is the default communication range of sensors andNum sensors is total number of sensors in network and Area is the area of sensing field.

\subsection{T-LEACH (Threshold Based LEACH)}

T-LEACH stands for threshold-based LEACH as itexchanges cluster heads of network depends on the threshold function value of residual energy on the nodes. Many type of clustering protocols are defined but defined clustering protocols do not focus on the energy-efficient interval of the cluster heads replacement. Cluster head election and substitution canunreasonablyscatter limited battery power of the whole sensor networks. T-LEACH reduces the number of cluster head election and substitution by using the threshold value of the residual energy. Minimizing the amount of head election and substitution cost, the lifespan of the whole networks can be prolonged as compared with the defined clustering protocols. 


\section{RELATED WORK}

Nasriet al. 2014[1] have presented a new approach for clustering based on Leach Protocol. This paper have discussed about the Leach protocol which is Low Energy Adaptive Clustering Hierarchy approach used to select nodes randomly as cluster head and distribute jobs according to some scheduling algorithm. Data transmission has been done by two phases known as setup phase and steady phase. In setup phase cluster heads are selected and in steady phase data is send to base station. The author of this paper proposed new approach called Leach-O that is a Static network in which cluster head send the data directly to the base station to avoid change of topology.LinaXuet al. 2014[2] have described an algorithm called BEEM (Balanced Energy Efficient Multihop) clustering. This Paper have analyzed different clustering algorithm. The algorithm BEEM exceeds the two clustering algorithm HEED and LEACH. The authors of paper have also defined the comparison of different clustering algorithm with the algorithm BEEM.The authors analyzed BEEM by using parameter of Cluster head rate, Network Distribution, clustering iteration, longevity and network coverage.Afsaret al. 2014[3] have described a state of art and comprehensive survey on the clustering algorithms. The authors have described the objectives of clustering and clustering characteristic. The authors have also described the steps to be followed in clustering process.Kuilaet al. 2014[4] have described Linear or non-linear programming formulations of the these problems followed by two proposed algorithms for the same based on articles warm optimization (PSO).The routing algorithm is developed with an efficient particle encoding scheme and multi objective functions.Xi Zhang $\boldsymbol{e t}$ al. 2013[5] have described mechanism of three dimensional clustering to maximize the life time of network by considering the minimum coverage rate constraint. This paper proposed new protocol by enhancing the leach protocol using the basic assumption of SEP protocol in which advanced nodes have more energy than initial node.Attarzadehet al. 2010[6] have describe the method of clustering called three dimensional clustering which resolve the restriction of two dimensional clustering by providing the different surface for review space. This paper define the three different levels, first for sink node which is at highest point, second level is fir cluster heads and third level is for different number of nodes which are active. This method is used to achieve better performance of network.Hong et al. 2008[7] have described the algorithm TLEACH. This protocol is based upon thresholdcluster head replacement technique for clustering algorithms of wireless sensor networks. T-LEACH protocolreduces the total number of cluster head collection by using threshold of residual energy. Authors simulation results have shown that T-LEACH beaten LEACH in terms of energy consumption balancing and in increasing lifespan of network.Chengzhiet al. 2008[8] have described the enhanced version of SEP protocol. SEP is stable election protocol that prolong the time interval before the death of first node. Sep is weighted election probabilities of each node to become a cluster head according to remaining energy in each node. In an Improved approach node with more energy will have more probability to become the cluster head node. This protocol is used to achieve maximum life time of network.Jennifer Yicket al. 2008 [9] have described a comprehensive review of the recent researches on wireless sensor network. The authors classify the problems into three different categories first is internal platform and underlying operating system, second is communication protocol stack, and third is network services, provisioning, and deployment. Authors review the major development in these three categories and outline new challenges. Authors of this paper have also summarized and compared different proposed designs,algorithms, protocols, and services.Srinathet al. 2007[10] have described the Authentication Confidentiality (AC) cluster-based secure routing protocol is three layer routing, where one layer is used to select cluster head, other layer for authentication and confidentiality and another layer is used for routing. Authors have presented the classification of routing techniques in wireless sensor network.Nasser $\boldsymbol{e t}$ al. 2006[11] have described comparison of different clustering protocols and algorithm. The authors of this paper have discussed about basic theoriesassociated to the clustering procedure, such as cluster organization, cluster natures, clustering techniques, and conciselyexamined LEACH-based protocols as well as proactive and reactive algorithms in WSN. The main features of these protocols are compared and the facts where they can be used now were defined. Loscret al 2005 [12] have Proposed new algorithm TL-LEACH which exceeds LEACH protocol. TL-LEACH use random rotation of local cluster base station and localized coordinate to enable scalability and robustness. The Authors have evaluated the performance with NS-2 and have observed that this protocol outperforms the leach protocol in terms of energy consumption and lifetime of network. Youniset al. 2004[13] have described HEED (Hybrid Energy Efficient Distributed) clustering algorithm. In this paper, Authors have defined HEED as a hierarchical, distributed, clustering scheme in which a single-hop communication pattern was retained within each cluster. The Cluster Head nodes were selected based upon two basic constraints such as residual energy and intra-cluster communication cost.Mhatreet al. 2004[14] have described the comparison of Homogeneous and heterogeneous sensor nodes.The Authors have proposed and examined a multi-hop alternative of LEACH that isknown as M-LEACH (Multi-hop Leach). The authors of paper have shown that MLEACH has improved energy efficiency than LEACH in many circumstances. The authors then compared the cost of multi-hop clustered sensor networks with M-LEACH as the typical homogeneous network, and a sensor network defined by two types of sensor nodes that practice intra-cluster multihopping as the illustrative heterogeneous network.Coyle $\boldsymbol{e t}$ al. 2003[15] have presented an Energy Efficient Clustering Hierarchy $(\mathrm{EECH})$ algorithm in which each sensor node in network become cluster head and advertised itself as the cluster head node to the sensor within its range. These cluster heads are known as volunteer cluster heads. Any sensor which is not volunteer $\mathrm{CH}$ or have not joined any cluster heads group, become cluster head and are called forced cluster head. This algorithm aims to find idealprospect of a node to turn out to become a cluster head. Heinzelman et al. 2002[16] have presented a protocol architecture for wireless networks. The authors of this paper described about the properties of sensor network which is used for the evaluation such as ease of deployment, system lifetime, latency, quality and introduced clustering protocol Leach-C based on leach protocol in which centralized clustering algorithm is used. Using central control algorithm to form the clusters may produce better cluster by dispersing the cluster head nodes throughout the network.P. Agarwalet al. 2002[17] have described Adaptive Threshold Energy Efficient Network (APTEEN) clustering algorithmto provide event based data communication. APTEEN is the improvement in TEEN and help for the responding periodically. APTEEN provides a TDMA-based structure for the communication of sensed message to the cluster and each sensor node send its information periodically to the respective $\mathrm{CH}$. Queries in APTEEN are defined by three types as Historical queries, One time queries and Persistent queries.Manjeshwaret al.2001[18] have described Threshold 
Energy Efficient Networkthat have two types of thresholds as Hard threshold and Soft threshold. In this protocol sensor nodes are programmed in such a way that it will react to sensed element changes by matching the estimated value to the HT, if HT exceeded, the sensor node transmit it observes data to the Cluster Head. ST is used to minimize the redundancy in the communication. Whenever the HT exceeded, the sensor node also finds the ST for resultant observations. Sensor node does not transmit this information, if the difference between consecutive observations does not exceed the ST.

\section{GAPS IN LITERATURE}

1. The use of the hard and soft thresholds has been ignored by the most of the existing researchers.

2. In BEEM a node might not become cluster head for a long time so will result in load misbalancing

3. The effect of the nodes scalability has been ignored in the BEEM.

\section{CONCLUSION AND FUTURE WORK}

Network lifetime is crucial in Wireless Sensor Network system, since recharging or exchanging the sensors is difficult and expensive. Clustering techniques provide an interface for WSN topology management to extend network lifetime. Existing clustering algorithms, such as LEACH and HEED, can significantly reduce the power consumption on each sensor and thus prolong the network lifetime. However, most existing work fails to consider the coverage of the network when evaluating the lifetime of a network. An advanced clustering algorithm should not only be able to extend the longevity, but also maintain the coverage. Although it is hard to achieve perfectly balanced load, the sensors that are still alive should be well distributed over the sensing area.

This work has not considered any improvement in the BEEM protocol. Therefore in near future, a novel algorithm BEEM clustering algorithm will be improved. The new algorithm will provide improvement in the BEEM by using the reactive principle i.e. hard and soft thresholds. In BEEM a node might not become cluster head for a long time so will result in load misbalancing so it will be removed by using the waiting nodes.

\section{REFERENCES}

[1] Khediri, Salim EL, et al. "A New Approach for Clustering in Wireless Sensors Networks Based on LEACH." Procedia Computer Science 32 (2014): 11801185.

[2] Xu, Lina, G. M. P. O'Hare, and Rem Collier. "A Balanced Energy-Efficient Multihop clustering scheme for Wireless Sensor Networks." Wireless and Mobile Networking Conference (WMNC), 2014 7th IFIP.IEEE, 2014.

[3] Afsar, M. Mehdi, and Mohammad-H.TayaraniN."Clustering in sensor networks: A literature survey."Journal of Network and Computer Applications (2014).

[4] Kuila, Pratyay, and Prasanta K. Jana. "Energy efficient clustering and routing algorithms for wireless sensor networks: Particle swarm optimization approach." Engineering Applications of Artificial Intelligence 33 (2014): 127-140.

[5] Xie, Lijun, and Xi Zhang. "3D clustering-based camera wireless sensor networks for maximizing lifespan with minimum coverage rate constraint." Global Communications Conference (GLOBECOM), 2013 IEEE.IEEE, 2013.

[6] Attarzadeh, Nima, et al. "Introduce methods for 3D clustering for wireless sensor networks." Electronics and Information Engineering (ICEIE), 2010 International Conference On.Vol.2.IEEE, 2010.

[7] Hong, Jiman, et al. "T-LEACH: The method of threshold-based cluster head replacement for wireless sensor networks." Information Systems Frontiers 11.5 (2009): 513-521.

[8] Long, Chengzhi, Lihong $\mathrm{Li}$, and Weiling Wu."An Improved Scheme of SEP in Heterogeneous Wireless Sensor Networks."Computational Intelligence and Industrial Application, 2008.PACIIA'08.Pacific-Asia Workshop on.Vol.1.IEEE, 2008.

[9] Yick, Jennifer, Biswanath Mukherjee, and DipakGhosal. "Wireless sensor network survey." Computer networks 52.12 (2008): 2292-2330.

[10] Srinath, Rampur, A. Vasudev Reddy, and R. Srinivasan. "Ac: Cluster based secure routing protocol for wsn." Networking and Services, 2007.ICNS.Third International Conference on.IEEE, 2007.

[11] Arboleda, Liliana MC, and Nidal Nasser."Comparison of clustering algorithms and protocols for wireless sensor networks."Electrical and Computer Engineering, 2006.CCECE'06.Canadian Conference on.IEEE, 2006.

[12] Loscri, V., G. Morabito, and S. Marano. "A two-levels hierarchy for low-energy adaptive clustering hierarchy (TL-LEACH)." IEEE Vehicular Technology Conference.Vol. 62.No. 3.IEEE; 1999, 2005.

[13] Younis, Ossama, and Sonia Fahmy. "HEED: a hybrid, energy-efficient, distributed clustering approach for ad hoc sensor networks." Mobile Computing, IEEE Transactions on 3.4 (2004): 366-379.

[14] Mhatre, Vivek, and Catherine Rosenberg. "Homogeneous vs heterogeneous clustered sensor networks: a comparative study." Communications, 2004 IEEE International Conference on.Vol.6.IEEE, 2004.

[15] Bandyopadhyay, Seema, and Edward J. Coyle. "An energy efficient hierarchical clustering algorithm for wireless sensor networks."INFOCOM 2003. TwentySecond Annual Joint Conference of the IEEE Computer and Communications. IEEE Societies.Vol. 3.IEEE, 2003.

[16] Heinzelman, Wendi B., Anantha P. Chandrakasan, and HariBalakrishnan."An application-specific protocol architecture for wireless microsensor networks." Wireless Communications, IEEE Transactions on 1.4 (2002): 660-670.

[17] Manjeshwar, Arati, and Dharma P. Agrawal. "APTEEN: A hybrid protocol for efficient routing and comprehensive information retrieval in wireless sensor networks." Parallel and Distributed Processing Symposium, International. Vol. 2.IEEE Computer Society, 2002.

[18] Manjeshwar, Arati, and Dharma P. Agrawal. "TEEN: a routing protocol for enhanced efficiency in wireless sensor networks." Parallel and Distributed Processing Symposium, International. Vol. 3.IEEE Computer Society, 2001. 